\title{
\begin{tabular}{l|l} 
Mitraries & DSpace@MIT
\end{tabular}
}

\author{
MIT Open Access Articles
}

Bearing Capacity of Spatially Random Cohesive Soil Using Numerical Limit Analyses

The MIT Faculty has made this article openly available. Please share how this access benefits you. Your story matters.

Citation: Kasama, Kiyonobu, and Andrew J. Whittle. “Bearing Capacity of Spatially Random Cohesive Soil Using Numerical Limit Analyses." Journal of Geotechnical and Geoenvironmental Engineering (2011)

As Published: http://dx.doi.org/10.1061/(ASCE)GT.1943-5606.0000531

Publisher: American Society of Civil Engineers

Persistent URL: http://hdl.handle.net/1721.1/67294

Version: Author's final manuscript: final author's manuscript post peer review, without publisher's formatting or copy editing

Terms of use: Creative Commons Attribution-Noncommercial-Share Alike 3.0 


\section{Bearing Capacity of Spatially Random Cohesive Soil Using Numerical Limit Analyses}

by

Kiyonobu Kasama ${ }^{1}$ and Andrew J. Whittle ${ }^{2}$, M.ASCE

ABSTRACT: This paper describes a probabilistic study of the two dimensional bearing capacity of a vertically loaded strip footing on spatially random, cohesive soil using Numerical Limit Analyses (NLA-CD). The analyses uses a Cholesky Decomposition (CD) technique with mid-point discretization to represent the spatial variation in undrained shear strength within finite element meshes for both upper and lower bound analyses, and assumes an isotropic correlation length. Monte Carlo simulations are then used to interpret the bearing capacity for selected ranges of the coefficient of variation in undrained shear strength and the ratio of correlation length to footing width. The results are compared directly with data from a very similar study by Griffiths et al. in which bearing capacity realizations were computed using a method of Local Average Subdivision (LAS) in a conventional displacement-based Finite Element Method (FEM-LAS). These comparisons show the same qualitative features, but suggest that the published FEM calculations tend to overestimate the probability of failure at large correlation lengths. The NLA method offers a more convenient and computationally efficient approach for evaluating effects of variability in soil strength properties in geotechnical stability calculations.

Keywords: Bearing capacity; cohesive soil, limit analysis; Monte Carlo method; Random field,

Probabilistic analysis

\footnotetext{
${ }^{1}$ Research Associate, Division of Civil and Structural Engineering, Faculty of Engineering, Kyushu University, Fukuoka, Japan.

2 Professor, Department of Civil and Environmental Engineering, Massachusetts Institute of Technology,
} 


\section{Introduction}

Recent numerical formulations of upper and lower bound limit analyses for rigid perfectly plastic materials, using finite element discretization and linear (Sloan, 1988a; Sloan \& Kleeman, 1995) or non-linear (Lyamin \& Sloan, 2002a, b) programming methods, provide a practical, efficient and accurate method for performing geotechnical stability calculations. For example, Ukritchon et al. (1998) proposed a solution to the undrained stability of surface footings on non-homogeneous and layered clay deposits under the combined effects of vertical, horizontal and moment loading to a numerical accuracy of $\pm 5 \%$. The only parameter used in these Numerical Limit Analyses, NLA, is the undrained shear strength (which can vary linearly within a given soil layer). Hence, NLA provides a more convenient method of analyzing stability problems than conventional displacement-based finite element methods which also require the specification of (elastic) stiffness parameters, simulation and interpretation of the complete non-linear load-deformation response up to collapse (e.g., Popescu et al., 2005).

This paper investigates a probabilistic approach to evaluating the bearing capacity of a planar footing on clay by incorporating the stochastic spatial variability of undrained shear strength within the numerical limit analyses. The undrained shear strength is treated as a random field (Vanmarcke, 1984) which is characterized by a log-normal distribution and a spatial correlation length (i.e., isotropic correlation structure). The current calculations use a Cholesky Decomposition technique with mid-point discretization (Baecher \& Christian, 2003; Matthies et al., 1997) to incorporate these random properties in numerical limit analyses (NLA-CD). The bearing capacity is then interpreted statistically from a series of Monte Carlo simulations.

Griffiths and Fenton (2001), Griffiths et al. (2002) and Popescu et al. (2005) have

Cambridge, MA02139. 
presented similar studies of the undrained bearing capacity of planar footings on clay using conventional displacement-based finite element analyses incorporating a linearly-elastic, perfectly plastic soil model (with deterministic elastic stiffness properties). The study by Popescu et al. (2005) uses the mid-point method for representing spatial variability of clays with non-Gaussian undrained shear strength properties (beta and gamma distribution), while Griffiths and Fenton (2001) has used a more rigorous method of Local Area Subdivision to represent spatial variability in the finite element model (FEM-LAS; after Fenton \& Vanmarcke, 1990) assuming log-normally distributed undrained shear strengths.

The current paper provides a completely independent method of evaluating undrained stability, but follows the statistical assumptions on clay shear strength properties and notations introduced by Griffiths et al. (2002) to facilitate the comparisons of results.

\section{Numerical Limit Analysis with Spatially Random Cohesive Soil}

Figures 1 illustrates a typical finite element mesh used to compute upper and lower bounds on the two dimensional bearing capacity of a vertically loaded plane strain footing of width, B. The lower bound analyses are based on the linear programming formulation presented by Sloan (1988a) and assume a linear variation of the unknown stresses $\left(\sigma_{\mathrm{x}}, \sigma_{\mathrm{y}}, \tau_{\mathrm{xy}}\right)$ within each triangular element. The formulation differs from conventional displacement-based finite-element formulations by assigning each node uniquely within an element, such that the unknown stresses are discontinuous along adjacent edges between elements. Statically admissible stress fields are generated by satisfying: i) a set of linear equality constraints, enforcing static equilibrium with triangular elements and along stress discontinuities between the elements, ii) inequality constraints that ensure no violation of the linearized material failure criterion. The current analyses assume 
a Tresca yield criterion for the undrained shear strength of clay. The lower-bound estimate of the collapse load is then obtained through an objective function that maximizes the resultant vertical force acting on the footing. The linear programming problem is solved efficiently using a steepest edge active set algorithm (Sloan, 1988b).

The upper-bound formulation also discretizes the soil mass into three-noded triangular elements, Figure 1, with linear variations in the unknown velocities $\left(u_{\mathrm{x}}, u_{\mathrm{y}}\right)$. Nodes are unique to each element and hence, the edges between elements represent planes of velocity discontinuities. Plastic volume strains and shear strain rates can occur within each element as well as along velocity discontinuities. The kinematic constraints are defined by the compatibility equations and the condition of associated flow (based on an appropriate linearization of the Tresca criterion) within each element and along the velocity discontinuities between elements. The external applied load can be expressed as a function of unknown nodal velocities and plastic multiplier rates. The upper-bound on the collapse load is then formulated as a linear programming problem, which seeks to minimize the external applied load using an active set algorithm (after Sloan \& Kleeman, 1995).

One of the principal advantages of NLA is that the true collapse load is always bracketed by results from the upper and lower bound calculations (for all materials obeying an associated flow rule). However, careful mesh refinement is essential in order to achieve numerically accurate solutions. Sloan (1988a) and Sloan \& Kleeman (1995) have reported the influence of mesh refinement and approximation of linearized Tresca criterion in the lower bound and upper bound numerical limit analyses respectively. Based on prior studies by Ukritchon et al. (1998), the current upper bound analyses use a uniform mesh with elements of characteristic dimension 0.125B, Figure 1. The size of the discretized domain is sufficient to contain all potential failure 
mechanisms, such that the far field boundaries can be represented as zero velocity conditions.

The current study uses a similar uniform mesh for the Lower Bound analyses in order to ensure a consistent interpretation of the sample functions of undrained shear strengths. However, extension elements are needed in the LB analyses to ensure that lower-bound conditions are rigorously satisfied in the far field. Prior studies (e.g., Ukritchon et al., 1998) have found that the accuracy of LB stress analyses can be improved by increasing mesh density close to singularities at the edge of the footing. The subsequent results show that such refinements are unnecessary for the current problem.

The effects of inherent spatial variability are represented in the analyses by modeling the undrained shear strength, $s_{u}$, as a homogeneous random field while the effect of the spatial variability of soil density is neglected by assuming the soil to be weightless. The undrained shear strength is assumed to have an underlying log-normal distribution with mean, $\mu_{s_{u}}$, and standard deviation, $\sigma_{s_{u}}$, and an isotropic scale of fluctuation (also referred to as the correlation length), $\theta_{\ln _{s}}$. The use of the log-normal distribution is predicated by the fact that $s_{u}$ is always a positive quantity. Phoon and Kulhawy (1999) have compiled data on the inherent variability of $s_{u}$ and report typical Coefficients of Variation in undrained shear strength, $C O V_{s_{u}}=\sigma_{s_{u}} / \mu_{s_{u}}=0.1-$ 0.8 , based on conventional laboratory shear tests. The mean and standard deviation of $\log s_{u}$ are readily derived from $C O V_{s_{u}}$ and $\mu_{s_{u}}$ as follows (e.g., Baecher \& Christian, 2003):

$$
\begin{aligned}
& \sigma_{\ln s_{u}}=\sqrt{\ln \left(1+C O V_{s_{u}}^{2}\right)} \\
& \mu_{\ln s_{u}}=\ln \mu_{s_{u}}-\frac{1}{2} \sigma_{\ln s_{u}}^{2}
\end{aligned}
$$

There are much fewer data available to evaluate the scale of fluctuation which 
corresponds to the physical distance over which there is correlation in the undrained shear strength. Although some studies have found that the horizontal scale of fluctuation can be an order of magnitude greater than the vertical scale (e.g., James Bay marine clay deposits; DeGroot \& Baecher, 1983), the local geological environment is likely to have a major influence on the correlation length parameter(s). Following Griffiths et al. (2002) the current analyses present results based on assumed values of the ratio of the correlation length to footing width, $\Theta_{\ln s_{u}}=\theta_{\ln s_{u}} / B$

The spatial variability is incorporated within the NLA meshes by assigning the undrained shear strength corresponding to the $i^{\text {th }}$ element:

$$
S_{u_{i}}=\exp \left(\mu_{\ln s_{u}}+\sigma_{\ln s_{u}} G_{i}\right)
$$

where $G_{i}$ is a random variable that is linked to the spatial correlation length, $\theta_{\ln s_{u}}$.

Values of $G_{i}$ are obtained using a Cholesky Decomposition technique (e.g., Baecher \& Christian, 2003) using an isotropic Markov function which assumes that the correlation decreases exponentially with distance between two points $i, j$ :

$$
\rho\left(x_{i j}\right)=\exp \left\{-\frac{2 x_{i j}}{\theta_{\ln s_{u}}}\right\}
$$

where $\rho$ is the correlation coefficient between two random values of $s_{u}$ at any points separated by a distance $x_{i j}=\left|\boldsymbol{x}_{i}-\boldsymbol{x}_{j}\right|$ where $\boldsymbol{x}_{i}$ is the position vector of $i$ (located at the center of element $i$ in the finite element mesh). This correlation function can be used to generate a correlation matrix, $\boldsymbol{K}$, which represents the correlation coefficient between each of the elements used in the NLA finite element meshes: 


$$
\boldsymbol{K}=\left[\begin{array}{cccc}
1 & \rho_{12} & \cdots & \rho_{1 n} \\
\rho_{12} & 1 & \cdots & \rho_{2 n} \\
\vdots & \vdots & \ddots & \vdots \\
\rho_{1 n} & \rho_{2 n} & \cdots & 1
\end{array} \mid\right.
$$

where $\rho_{i j}$ is the correlation coefficient between element $i$ and $j$, and $n$ the total number of elements in the mesh.

The matrix $\boldsymbol{K}$ is positive definite and hence, the standard Cholesky Decomposition algorithm can be used to factor the matrix into triangular forms used in NLA mesh, $S$ and $\boldsymbol{S}^{\mathrm{T}}$, respectively:

$$
S^{T} S=K
$$

The components of $S^{T}$ are specific to a given finite element mesh and selected value of the correlation length, $\theta_{\ln s_{u}}$.

The vector of random variables, $\boldsymbol{G}$ (i.e., $\left\{G_{1}, G_{2}, \cdots, G_{n}\right\}$, where $G_{i}$ specifies the random component of the undrained shear strength in element $i$, eqn. 3) can then be obtained from the product:

$$
G=S^{T} X
$$

where $\boldsymbol{X}$ is a vector of statistically independent, random numbers $\left\{x_{1}, x_{2}, \cdots, x_{n}\right\}$ with a standard normal distribution (i.e., with zero mean and unit standard deviation).

The current implementation implicitly uses the distance between the centroids to define the correlation between undrained shear strengths in adjacent elements. This is an approximation of the random field, which involves the integral of the correlation function over the areas of the two elements. Figure 2 compares the exponential function for four correlation lengths with results estimated from a single realization obtained using the proposed mid-point $\mathrm{CD}$ technique 
for the UB mesh shown in Figure 1. The data show good agreement with the correlation function for intervals as small as $0.05 \mathrm{~B}$, corresponding to the minimum distance between the centroids of adjacent elements. The results suggest that the current mesh can provide an adequate representation for correlation lengths, $\Theta_{\ln s_{u}} \geq 0.1$.

Values of the random variable vector $\boldsymbol{X}$ are then re-generated for each realization in a set of Monte Carlo simulations. Figure 1 illustrates the spatial distribution of undrained shear strength obtained for typical mesh for one example simulation with input parameters $\mu_{s_{u}}$ $=100 \mathrm{kPa}, C O V_{c_{u}}=0.2$ and $\Theta_{\ln s_{u}}=1.0 . \quad$ The lighter shaded regions indicate areas of higher shear strength.

\section{Bearing Capacity Results}

Upper and lower bound stability calculations have been performed assuming a fixed mean value for the undrained shear strength, $\mu_{s_{u}}=100 \mathrm{kPa}$, while varying combinations of the coefficient of variation and correlation length over the following ranges:

$$
\begin{aligned}
& C O V_{s_{u}}=0.2,0.4,0.6,0.8,1.0,4.0 \\
& \Theta_{\ln s_{u}}=0.1,0.2,1.0,2.0,4.0,8.0,20
\end{aligned}
$$

Figure 3 illustrates the effects of the correlation length parameter on the mechanisms of failure from a series of three UB simulations with $C O V_{s_{u}}=0.4$ and $\Theta_{\ln s_{u}}=0.2,1.0,2.0$. Each example shows the specific realization of the undrained strength field superimposed on the deformed FE mesh, together with the vectors of the computed velocity field (dark shaded regions in these figures represent locations where plastic distortion occurs within the finite elements). The strength field appears ragged for $\Theta_{\ln s_{u}}=0.2$ but is much smoother for $\Theta_{\ln s_{u}}=1.0,2.0$. 
Close inspection shows that the computed failure mechanisms find paths of least resistance, passing through weaker regions of the clay.

A series of 1000 Monte Carlo simulations have been performed for each combination of the input parameters $\left(C O V_{s_{u}}, \Theta_{\ln _{s}}\right)$. The computed bearing capacity factor, $N_{c i}$, can then be reported for each realization of the shear strength field:

$$
N_{c i}=q_{f i} / \mu_{s_{u}}, \quad \text { where } i=1,2, \ldots n \ldots 1000
$$

where $q_{f i}$ is the computed collapse load (either UB or LB).

The mean, $\mu_{N_{c}}$, and standard deviation, $\sigma_{N_{c}}$, of the bearing capacity factor are recorded through each set of Monte Carlo simulations, as follows:

$$
\mu_{N_{c}}=\frac{1}{n} \sum_{i=1}^{n} N_{c i} ; \quad \sigma_{N_{c}}=\sqrt{\frac{1}{n-1} \sum_{i=1}^{n}\left(N_{c i}-\mu_{N_{c}}\right)^{2}} .
$$

Figure 4 illustrates one set of results for the case with $\Theta_{\ln s_{u}}=2.0, C O V_{s_{u}}=0.2$ and 0.8 .

The results confirm that the collapse load for any given realization is well bounded by $\mu_{N_{c}}$ from the UB and LB calculations. The mean and standard deviation of $N_{c}$ become stable within a few hundred simulations.

Table 1 summarizes the statistical data for the bearing capacity factor for all combinations of the input parameters. In all cases the results show $\mu_{N_{c}}(U B)>\mu_{N_{c}}(L B)$, and the actual collapse load is typically bounded within $\pm 5-10 \%$ showing acceptable accuracy from the numerical limit analyses. The data also show $\sigma_{N_{c}}(U B)>\sigma_{N_{c}}(L B)$. This latter result may reflect differences in the upper bound and lower bound limit analyses. However, it is notable that the numerical limit analyses generate much smaller coefficients of variation in bearing capacity than were reported by Griffiths et al. (2002) from FEM-LAS simulations (the data in 
Table 1 show $\left.C O V_{N_{c}}=\sigma_{N_{c}} / \mu_{N_{c}}=0.03-1.16\right)$.

Figure 5 presents a 20-bin histogram of the bearing capacity factor from one complete series of Monte Carlo simulations with $C O V_{s_{u}}=0.2$ and $\Theta_{\ln s_{u}}=2.0$ together with the estimated normal distribution. In order to obtain the distribution function of the bearing capacity factor based on $\chi^{2}$ goodness-of-fit tests, Table 1 summarizes $\chi^{2}$ statistics for all of the simulations and confirms that normal or log-normal distribution functions can be used to characterize the bearing capacity at a 5\% significance level (with acceptance level, $\left.\chi_{20-1-2}^{2}[0.05]=27.6\right)$.

Figures $6 \mathrm{a}$ and $6 \mathrm{~b}$ summarize the ratio of the mean bearing capacity factor to the deterministic solution for homogeneous clay, $\tilde{N}_{c}=\mu_{N_{c}} / N_{c \text { Det }}$ (where $N_{c \text { Det }}=[2+\pi]$ ) for combinations of the input parameters $\left(C O V_{s_{u}}, \Theta_{\ln s_{u}}\right)$. In general, $\tilde{N}_{c}<1$ and hence spatial variability causes a reduction in the expected undrained bearing capacity. The trends show that the largest reductions in $\mu_{N_{c}}$ occur when the coefficient of variation is high and/or the correlation length is small. Assuming a maximum realistic range, $C O V_{s_{u}} \leq 0.6-0.8$, the results suggest that the expected bearing capacity could be as little as $60 \%$ of the deterministic value.

Qualitatively similar results have been presented by Griffiths et al. (2002). However, these Authors also report a local minimum in the expected bearing capacity for $\Theta_{\ln s_{u}} \approx 1.0$, which is not seen in the current numerical limit analyses (Fig. 6b). The current analyses do show a widening gap between LB and UB solutions for $\Theta_{\ln s_{u}}<1.0$ (i.e., loss of accuracy), which reflects the underlying problem of stochastic discretization that requires elements to be smaller than the spatial correlation length (e.g., Matthies et al., 1997). As a result, the current NLA-CD analyses do not converge to the theoretical limits as $\Theta_{\ln s_{u}} \rightarrow 0$. 


\section{Probability of Failure}

In conventional working stress design practice an average undrained shear strength is used to compute the ultimate bearing capacity, while the allowable/nominal load is then obtained by applying a global safety factor, $F S=2.0-3.0$. In the current calculations the probability that the bearing capacity is less than a given level of applied load can be obtained by assuming that $N_{c}$ can be described by either a normal or log-normal distribution (as shown in Table 1). If $N_{c}$ is log-normally distributed, the probability that the bearing capacity is less than the nominal load is given by:

$$
P\left[N_{c}<N_{c D e t} / F S\right]=\Phi\left(\frac{\ln ([2+\pi] / F S)-\mu_{\ln N_{c}}}{\sigma_{\ln N_{c}}}\right)
$$

where $\Phi(.$.$) is the cumulative normal function and values of \mu_{\ln N_{c}}, \sigma_{\ln N_{c}}$ are reported from the NLA-CD analyses in Table 1.

Figure 7 summarizes predictions that the probability of bearing failure is less than the nominal load level for $F S=1.0,2.0$ and 3.0 as functions of the coefficient of variation in undrained shear strength, $C O V_{s_{u}}$, for correlation length parameters, $\Theta_{\ln s_{u}}=1.0,2.0$ and 4.0. As expected, when spatial variability is included in the analyses, the ultimate bearing capacity is almost always less than the deterministic capacity based on the mean shear strength. These results agree with earlier solutions from FEM-LAS reported by Griffiths et al. (2002) (for the same range of input parameters).

The probability that the bearing capacity is less than the nominal design load for $F S=2.0$ and 3.0 decreases very markedly with the coefficient of variation in undrained shear strength, especially for $C O V_{s_{u}}<1.0$, Figure 7, and also with increasing values of the spatial correlation 
length ratio, $\Theta_{\ln s_{u}}$.

For $\Theta_{\ln s_{u}}=1.0$, the UB predictions of the probability, $P\left[N_{c}<N_{c D e l} / F S\right]$ are in excellent agreement with prior data presented by Griffiths et al. (2002). However, the current analyses show lower event probabilities for correlation length ratios, $\Theta_{\ln s_{u}}=2.0,4.0$. The source of this discrepancy is not obvious and deserves further investigation.

Figure 8 offers a more detailed comparison of the probability of bearing failure implicitly defined in conventional design methods with the actual probabilities of failure derived from the stochastic NLA-CD analyses accounting for inherent spatial variability. The figures plot the $P\left[N_{c}<N_{c D e l} / F S\right]$ as functions of the safety factor, $F S$ for selected ranges of the input parameters $\operatorname{COV}_{s_{u}}$ and $\Theta_{\ln s_{u}}$. The target probabilities of failure considered in LRFD codes for shallow foundations are reported in the range, $P_{f}=10^{-2}-10^{-3}$ (Baecher \& Christian, 2003; Phoon et al., 2000). The results in Figure $8 \mathrm{a}$ show that $P\left[N_{c}<N_{c D e} / F S\right]$ is much less than this target condition for small values of the coefficient of variation, $C O V_{s_{u}}=0.2$. There is close agreement between the conventional working stress design and LFRD methods for $C O V_{s_{u}}=0.4,0.6$, Figures 8b, c. However, in exceptional cases with $\operatorname{COV}_{s_{u}}=0.8$ and $\Theta_{\ln s_{u}} \leq 1.0$, the estimated probability of failure can exceed $P_{f}=10^{-2}$ at $F S=3.0$.

\section{Conclusions}

This paper summarizes the implementation of a mid-point Cholesky Decomposition method for representing inherent spatial variability of undrained shear strength in Monte Carlo simulations of bearing capacity for a rough, surface strip footing on clay using Numerical Limit Analyses (NLA-CD). Accurate estimates of the exact bearing capacity are achieved in each 
Monte-Carlo realization. The analyses assume that undrained shear strength is described by a log-normal distribution function, while effects of spatial variability are characterized by two input parameters, i) the coefficient of variation, $C O V_{s_{u}}$ and ii) an isotropic correlation length ratio, $\Theta_{\ln s_{u}}$. Stable bearing capacity statistics were derived from a series 1000 Monte Carlo simulations for each set on input parameters. The current parametric calculations are then compared with results from a similar study reported by Griffiths et al. (2002) using a completely independent method of analysis (FEM-LAS).

The results confirm that spatial variability reduces the bearing capacity of the footing relative to a deterministic calculation based on the mean undrained shear strength. This result occurs due to changes in the predicted failure mechanisms which form through weaker regions in the clay. The lowest values in the computed ratio, $\tilde{N}_{c}=\mu_{N_{c}} / N_{c D e t}$, occur at high values of $C O V_{s_{u}}$ and small correlation length ratios $\left(\Theta_{\ln s_{u}}<1\right)$ in this analyses.

Although there is very good qualitative agreement with results presented by Griffiths et al. (2002) the current analyses generally suggest lower probabilities of design failure for the same input properties of the undrained shear strength field. This result will require further investigation through direct comparison of stochastic NLA and FEM methods.

The results suggest that target probabilities for bearing failure in the range $P_{f}=10^{-2}-$ $10^{-3}$ are consistent with conventional working stress design methods using $F S=2.0-3.0$ except in cases where there is very high coefficient of variation, $C O V_{s_{u}} \geq 0.8$ and/or small correlation ratios, $\Theta_{\ln s_{u}}<1$. 


\section{Acknowledgment}

The Authors are grateful to the Japanese Ministry of Education (MEXT) for providing Post-Doctoral support to the first Author at MIT. The Authors are also grateful to Dr John Christian for his detailed review and advice in editing this paper.

\section{References}

Baecher, G.B. and Christian, J.T. (2003). Reliability and statistics in geotechnical engineering, John Wiley \& Sons, Ltd., NY, 605p.

DeGroot, D.J. and Baecher, G.B. (1983). "Estimating autocovariance of in situ soil properties," ASCE J. Geotech. Eng., 129(1), 147-166.

Fenton, G. A., and Vanmarcke, E. H. (1990). "Simulation of random fields via local average subdivision."' J. Eng. Mech., 116(8), 1733-1749.

Griffiths, D. V., and Fenton, G. A. (2001). "Bearing capacity of spatially random soil: The undrained clay Prandtl problem revisited." Géotechnique, 51(4), 351-359.

Griffiths, D. V., Fenton, G. A., and Manoharan, N. (2002). "Bearing capacity of rough rigid strip footing on cohesive soil: probabilistic study.” ASCE J. Geotech. Eng., 128(9), 743-755.

Lyamin, A.V. and Sloan, S.W. (2002a). "Lower bound limit analysis using non-linear programming," Intl. Journal for Numerical Methods in Engineering, 55(5), 573-611.

Lyamin, A.V. and Sloan, S.W. (2002b). "Upper bound limit analysis using finite elements and non-linear programming," Intl. Journal for Numerical and Analytical Methods in Geomechanics, 26(2), 181-216.

Matthies, H.G., Brenner, C.E., Bucher, C.G. and Guedes Soares, C. (1997). 'Uncertainties in probabilistic numerical analysis of structures and solids - Stochastic finite elements," 
Structural Safety, 19(3), 283-336.

Phoon, K.K. and Kulhawy, F.H. (1999). "Characterization of geotechnical variability," Canadian Geotechnical Journal, 36, 612-624.

Phoon, K.K., Kulhawy, F.H. and Grigoriu, M.D. (2000). "Reliability-based design for transmission line structure foundations," Computers \& Geotechnics, 26, 169-185.

Popescu, R., Deodatis, G. and Nobahar, A. (2005). "Effects of random heterogeneity of ssoil properties on bearing capacity," Probabilistic Engineering Mechanics, 20, 324-341.

Sloan, S. W. (1988a). "Lower bound limit analysis using finite elements and linear programming." Int. J. Numer. Analyt. Meth. Geomech., 12(1), 61-77.

Sloan, S. W. (1988b). "A steepest edge active set algorithm for solving sparse linear programming problems." Int. J. Numer. Analyt. Meth. Geomech., 12(12), 2671-2685.

Sloan, S. W., and Kleeman, P. W. (1995). "Upper bound limit analysis using discontinuous velocity fields." Comput. Methods Appl. Mech. Eng., 127, 293-314.

Ukritchon, B., Whittle, A. J., and Sloan, S. W. (1998). "Undrained limit analyses for combined loading of strip footing on clay." J. Geotech. Eng., 124(3), 265-276.

Vanmarcke, E. H. (1984). Random fields: Analysis and synthesis. MIT Press, Cambridge, Mass. 


\section{NOTATION}

$B \quad$ = width of foundation;

$C O V_{s_{u}}=$ coefficient of variation of undrained shear strength;

$s_{u} \quad=$ undrained shear strength;

$s_{u_{i}} \quad=$ undrained shear strength of $i$ th element;

$F S \quad=$ safety factor;

$G(x) \quad=$ standard Gaussian field with zero mean unit variance;

$G\left(x_{i}\right) \quad=$ local value of standard Gaussian field with zero mean unit variance for $i$ th element;

$N_{c} \quad=$ bearing capacity factor;

$N_{c_{i}} \quad=$ bearing capacity factor for $i^{\text {th }}$ realization;

$P[\ldots] \quad=$ probability;

$u_{x}, u_{y} \quad=$ velocity components in $\mathrm{x}$ and $\mathrm{y}$ directions

$\boldsymbol{x}_{i} \quad=$ position vector at center of $i^{\text {th }}$ element;

$\Theta_{\ln s_{u}}=\theta_{\ln s_{u}} / B$, dimensionless correlation length ratio,

$\theta_{\ln s_{u}} \quad=$ spatial correlation length;

$\mu_{s_{u}} \quad=$ mean undrained shear strength;

$\mu_{\ln s_{u}} \quad=$ mean of log undrained shear strength;

$\mu_{\ln N_{c}}=$ mean of $\log$ bearing capacity factor;

$\mu_{N_{c}} \quad=$ mean bearing capacity factor;

$\rho \quad=$ correlation coefficient;

$\sigma_{s_{u}} \quad=$ standard deviation of undrained shear strength; 
$\sigma_{\ln s_{u}} \quad=$ standard deviation of log undrained shear strength;

$\sigma_{\ln N_{c}}=$ standard deviation of log bearing capacity factor;

$\sigma_{N_{c}} \quad=$ standard deviation of bearing capacity factor;

$\Phi(\ldots) \quad=$ cumulative normal function. 
Journal of Geotechnical and Geoenvironmental Engineering. Submitted October 12, 2007; accepted March 8, 2011; posted ahead of print March 10, 2011. doi:10.1061/(ASCE)GT.1943-5606.0000531

Table 1. Bearing capacity factor statistics and goodness of fit results for normal and log-normal distribution

\begin{tabular}{|c|c|c|c|c|c|c|c|c|c|c|c|c|c|}
\hline \multirow{2}{*}{$\Theta_{\ln s_{u}}$} & \multirow{2}{*}{$C O V_{c_{u}}$} & \multicolumn{6}{|c|}{ LB } & \multicolumn{6}{|c|}{ UB } \\
\hline & & $\mu_{N_{c}}$ & $\sigma_{N_{c}}$ & $\chi^{2}$ & $\mu_{\ln N_{c}}$ & $\sigma_{\ln N_{c}}$ & $\chi^{2}$ & $\mu_{N_{c}}$ & $\sigma_{N_{c}}$ & $\chi^{2}$ & $\mu_{\ln N_{c}}$ & $\sigma_{\ln N_{c}}$ & $\chi^{2}$ \\
\hline \multirow{6}{*}{0.1} & 0.2 & 4.330 & 0.082 & 22.2 & 1.465 & 0.019 & 24.3 & 4.815 & 0.099 & 27.1 & 1.572 & 0.021 & 22.3 \\
\hline & 0.4 & 3.572 & 0.127 & 20.2 & 1.272 & 0.036 & 19.2 & 4.16 & 0.171 & 24.5 & 1.425 & 0.041 & 24.2 \\
\hline & 0.6 & 2.858 & 0.154 & 24.0 & 1.049 & 0.054 & 27.2 & 3.472 & 0.217 & 22.8 & 1.243 & 0.062 & 27.3 \\
\hline & 0.8 & 2.353 & 0.145 & 21.3 & 0.854 & 0.061 & 25.1 & 2.937 & 0.208 & 22.3 & 1.075 & 0.07 & 15.1 \\
\hline & 1.0 & 1.921 & 0.157 & 14.2 & 0.649 & 0.084 & 10.4 & 2.467 & 0.238 & 22.3 & 0.898 & 0.099 & 24.1 \\
\hline & 4.0 & 0.375 & 0.070 & 22.3 & -0.998 & 0.192 & 22.8 & 0.555 & 0.119 & 17.8 & $\begin{array}{l}-0.613 \\
\end{array}$ & 0.224 & 11.9 \\
\hline \multirow{6}{*}{0.2} & 0.2 & 4.425 & 0.108 & 24.9 & 1.487 & 0.024 & 21.2 & 4.821 & 0.126 & 22.4 & 1.573 & 0.026 & 26.4 \\
\hline & 0.4 & 3.737 & 0.178 & 27.4 & 1.317 & 0.047 & 16.6 & 4.215 & 0.225 & 14.4 & 1.437 & 0.053 & 24.5 \\
\hline & 0.6 & 3.053 & 0.257 & 20.8 & 1.112 & 0.085 & 16.7 & 3.512 & 0.375 & 18.7 & 1.250 & & 24.2 \\
\hline & 0.8 & 2.545 & 0.247 & 21.2 & 0.929 & & 26.1 & 2.986 & 45 & 21.2 & 87 & 17 & 23.7 \\
\hline & 1.0 & 2.146 & 0.257 & 26.8 & 0.756 & 0.126 & 19.3 & 2.580 & 0.352 & 26.7 & 0.938 & 0.145 & 14.7 \\
\hline & 4.0 & 0.449 & 0.112 & 26.7 & -0.835 & 0.268 & 22.8 & 0.591 & 0.168 & 13.7 & -0.570 & 0.308 & 15.8 \\
\hline \multirow{6}{*}{1} & 0.2 & $\begin{array}{l}4.617 \\
\end{array}$ & 0.238 & $\begin{array}{l}14.4 \\
\end{array}$ & $\begin{array}{l}1.528 \\
\end{array}$ & $\begin{array}{c}0.052 \\
\end{array}$ & $\begin{array}{c}15.1 \\
\end{array}$ & 4.788 & $\begin{array}{c}0.301 \\
\end{array}$ & 25.3 & 1.564 & $\begin{array}{c}0.063 \\
\end{array}$ & 25.2 \\
\hline & 0.4 & 4.033 & 0.512 & 21.5 & 1.386 & 0.132 & 20.3 & 4.187 & 0.584 & 26.5 & 1.422 & 0.144 & 27.2 \\
\hline & 0.6 & 3.541 & 0.568 & 15.1 & 1.250 & 0.173 & 24.8 & 3.701 & 0.645 & 22.7 & 1.292 & 0.191 & 20.7 \\
\hline & 0.8 & 3.155 & 0.589 & 14.5 & 1.127 & & 26.2 & & 0.682 & 12.9 & 1.148 & & 21.0 \\
\hline & 1.0 & 2.721 & 0.722 & 21.1 & 0.958 & 0.312 & 17.9 & 2.807 & 0.833 & 15.0 & 0.979 & 0.349 & 17.7 \\
\hline & 4.0 & 0.877 & 0.589 & 27.6 & -0.405 & 0.730 & 21.0 & 0.899 & 0.663 & 25.1 & -0.346 & 0.809 & 19.0 \\
\hline \multirow{6}{*}{2} & 0.2 & $\begin{array}{l}4.731 \\
\end{array}$ & 0.260 & 22.7 & 1.553 & 0.056 & $\begin{array}{l}15.0 \\
\end{array}$ & 4.860 & 0.307 & 16.2 & 1.579 & $\overline{c 0.064}$ & 20.4 \\
\hline & 0.4 & 4.278 & 0.476 & 15.7 & 1.447 & 0.120 & 26.8 & 4.342 & 0.539 & 25.1 & 1.460 & 0.132 & 15.2 \\
\hline & 0.6 & 3.785 & 0.609 & 24.1 & 1.317 & 0.175 & 24.4 & 3.823 & 0.722 & 21.3 & 1.321 & 0.205 & 20.4 \\
\hline & 0.8 & 3.418 & 0.758 & 18.4 & 1.202 & & 26.4 & 3.457 & 0.860 & 17.9 & 1.206 & 0.275 & 26.2 \\
\hline & 1.0 & 3.102 & 0.849 & 12.2 & 1.084 & & 22. & 3.137 & 0.935 & 11.2 & 1.104 & 0.318 & 18.7 \\
\hline & 4.0 & 1.417 & 0.805 & 27.1 & 0.126 & 0.644 & 21.9 & 1.503 & 0.899 & 26.7 & 0.230 & 0.710 & 16.7 \\
\hline \multirow{6}{*}{4} & 0.2 & 4.825 & 0.185 & 25.0 & 1.573 & 0.038 & 25.0 & \begin{tabular}{|l|}
4.940 \\
\end{tabular} & 0.225 & 99.7 & 1.596 & 0.046 & 10.6 \\
\hline & 0.4 & 4.522 & 0.369 & 21.1 & 1.506 & 0.083 & 22.7 & 4.605 & 0.461 & 21.4 & 1.522 & 0.103 & 14.3 \\
\hline & 0.6 & 4.235 & 0.512 & 18.6 & 1.436 & 0.125 & 21.5 & 4.318 & 0.606 & 20.4 & 1.452 & 0.146 & 23.3 \\
\hline & 0.8 & 3.698 & 0.726 & 23.5 & 1.280 & & 24 & 3.703 & 0.822 & 20.2 & 1.288 & 0. & 24.4 \\
\hline & 1.0 & & 0.801 & 20.6 & & & 21 & & 0.904 & 25.3 & & 9 & 25.8 \\
\hline & 4.0 & 1.958 & 0.938 & 22.1 & 0.512 & 0.576 & 21.6 & 1.976 & 0.973 & 22.3 & 0.541 & 0.627 & 22.9 \\
\hline \multirow{6}{*}{8} & 0.2 & 4.894 & 0.170 & 20.6 & 1.587 & 0.035 & $\begin{array}{l}15.0 \\
\end{array}$ & 5.022 & 0.189 & $\begin{array}{l}18.9 \\
\end{array}$ & 1.613 & 0.038 & 21.1 \\
\hline & 0.4 & 4.676 & 0.317 & 25.6 & 1.540 & 0.069 & 18.6 & 4.739 & 0.399 & 19.2 & 1.552 & 0.087 & 21.9 \\
\hline & 0.6 & 4.432 & 0.431 & 24.8 & 1.484 & 0.099 & 18.7 & 4.485 & 0.534 & 21.5 & 1.494 & 0.121 & 12.1 \\
\hline & 0.8 & 4.140 & 0.652 & 17.4 & 1.407 & & 24.8 & 4.200 & 0.77 & 15.2 & 1.417 & 0.195 & 16.2 \\
\hline & 1.0 & & 0.610 & 21.9 & & & 14.1 & & 0.721 & 14.7 & 1.343 & 0.193 & 18.7 \\
\hline & 4.0 & 2.773 & 0.925 & 16.4 & 0.936 & 0.388 & 19.6 & 2.817 & 1.034 & 19.2 & 0.970 & 0.441 & 14.8 \\
\hline \multirow{6}{*}{20} & 0.2 & 4.938 & 0.113 & 8.2 & 1.597 & 0.023 & 9.9 & 5.085 & 0.137 & 25.0 & 1.626 & 0.027 & 26.5 \\
\hline & 0.4 & 4.845 & 0.226 & 20.6 & 1.577 & 0.047 & 24.8 & 4.945 & 0.278 & 27.5 & 1.597 & 0.056 & 25.2 \\
\hline & 0.6 & 4.688 & 0.312 & 26.0 & 1.543 & 0.068 & 21.7 & 4.762 & 0.392 & 23.3 & 1.557 & 0.082 & 14.0 \\
\hline & 0.8 & 4.530 & 0.381 & 7.9 & 1.507 & 0.085 & 16.5 & 4.584 & 0.470 & 25.1 & 1.517 & 0.102 & 24.0 \\
\hline & 1.0 & 4.454 & 0.431 & 13.2 & 1.489 & 0.101 & 15.9 & 4.506 & 0.511 & 10.8 & 1.499 & 0.113 & 15.5 \\
\hline & 4.0 & 3.468 & 0.783 & 16.7 & 1.209 & 0.235 & 26.9 & 3.503 & 0.880 & 18.7 & 1.227 & 0.250 & 17.8 \\
\hline
\end{tabular}

Note: Acceptance criterion $\chi^{2}{ }_{20-1-2}[0.05] \leq 27.6$ 


\section{LIST OF FIGURES}

Fig. 1. Typical finite element mesh used in Upper Bound numerical limit analyses for rough strip footing $\left(C O V_{s_{u}}=0.2, \Theta_{\ln s_{u}}=1\right)$

Fig. 2. Comparison of exact correlation and realized correlation obtained by proposed mid-point Cholesky Decomposition technique

Fig. 3. Typical results of Upper Bound numerical limit analyses

Fig. 4. Summary statistics for bearing capacity factor as functions of the number of Monte Carlo simulations a) Mean bearing capacity factor; b) Standard deviation of bearing capacity factor

Fig. 5. Histogram and estimated normal distribution of bearing capacity factor

Fig. 6. Summary of mean bearing capacity ratio $\tilde{N}_{c}$

Fig. 7. Probability that the bearing capacity factor is less than the nominal design load
a) $\Theta_{\ln s_{u}}=1$; b) $\Theta_{\ln s_{u}}=2$; c) $\Theta_{\ln s_{u}}=4$

Fig. 8. Comparison of failure probability and conventional safety factor a) $\quad C O V_{s_{u}}=0.2$;

b) $C O V_{s_{u}}=0.4 ;$ c) $C O V_{s_{u}}=0.6$; d) $C O V_{s_{u}}=0.8$ 


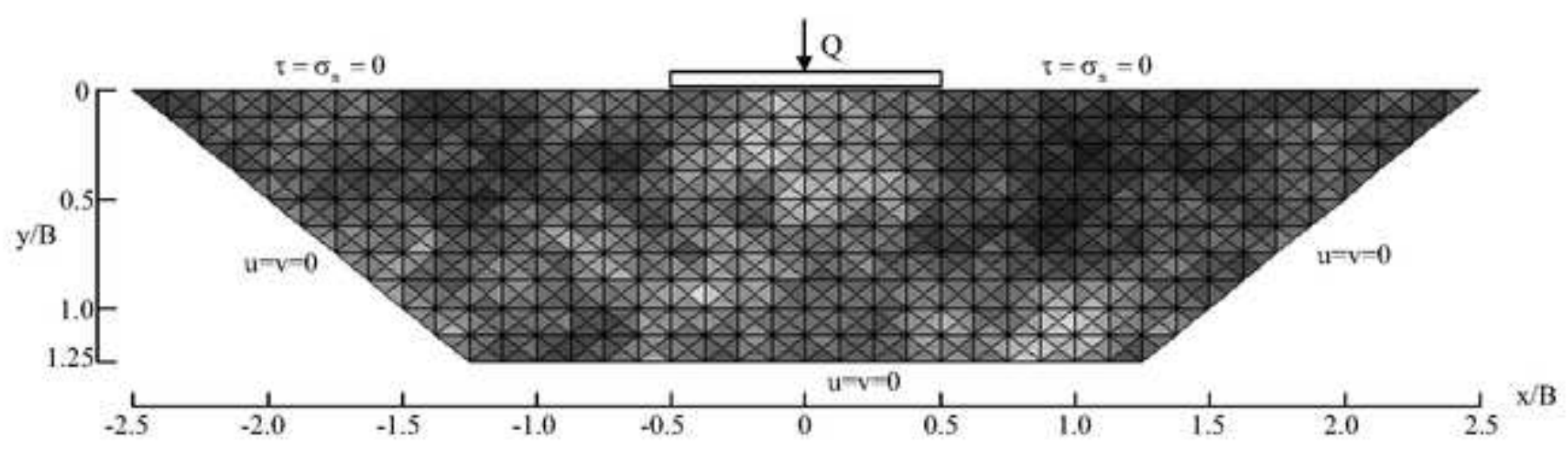

Fig. 1. Typical finite element mesh used in Upper Bound numerical limit analysis for rough strip footing $\left(\mathrm{COV}_{s_{\mathrm{n}}}=0.2, \Theta_{\ln s_{s}}=1\right)$ 


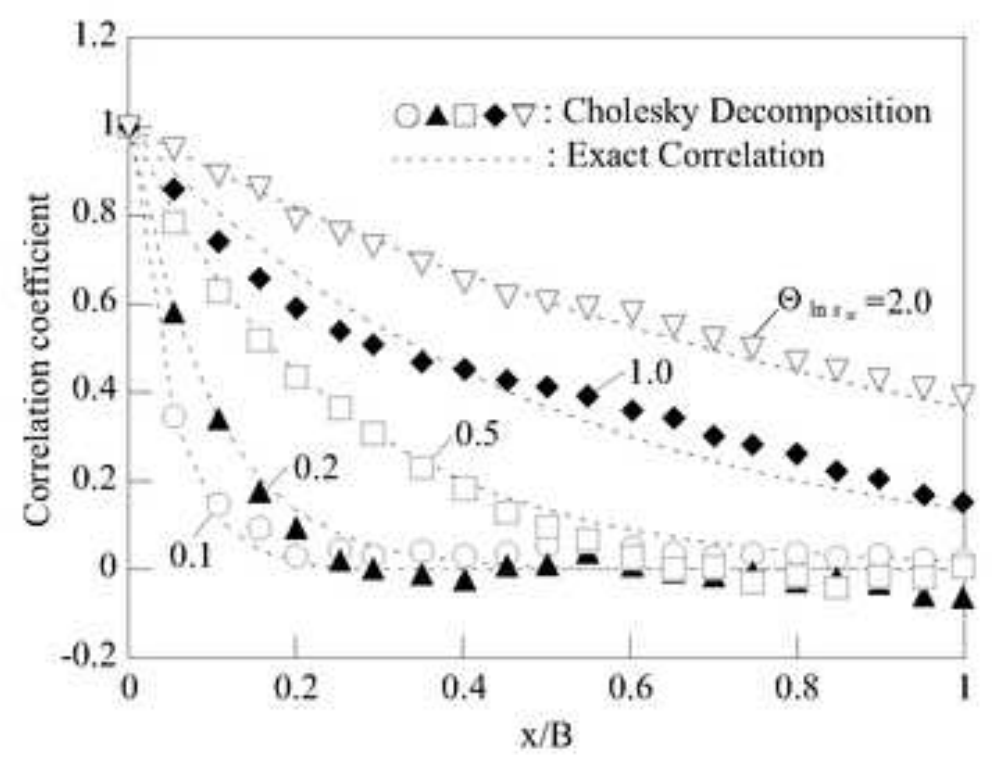

Fig. 2. Comparison of exact correlation relation and realized correlation obtained by proposed mid-point Cholesky Decomposition technique 

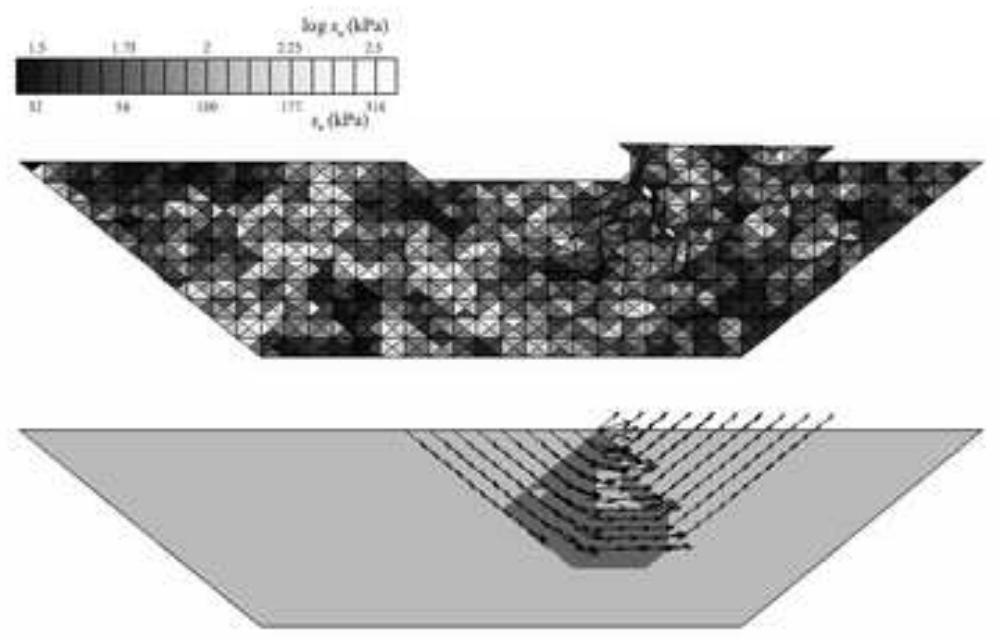

a) $\operatorname{COV}_{x_{s}=0.4,} \Theta_{\underline{m s}=0.2}$
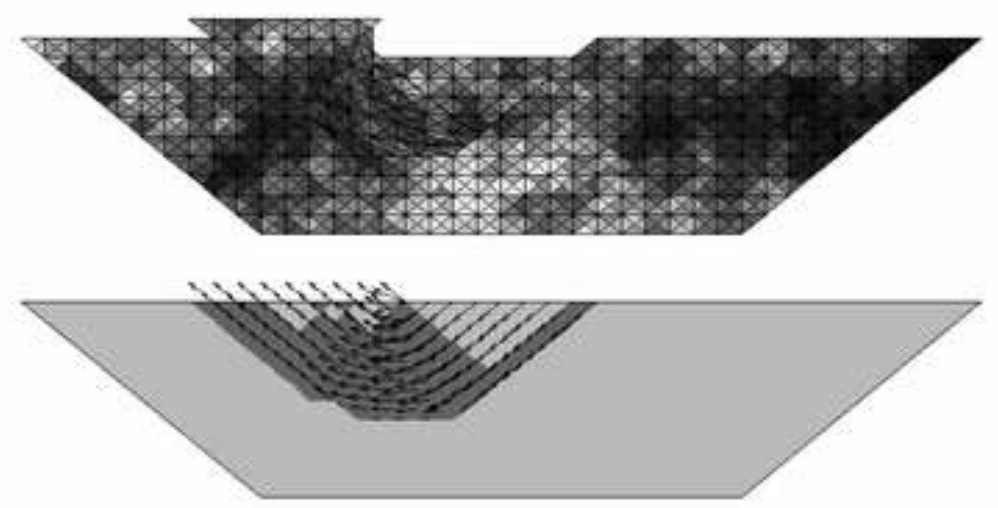

b) $\operatorname{COV}_{s_{\alpha}=0.4,} \Theta_{\ln x_{\alpha}=1}$
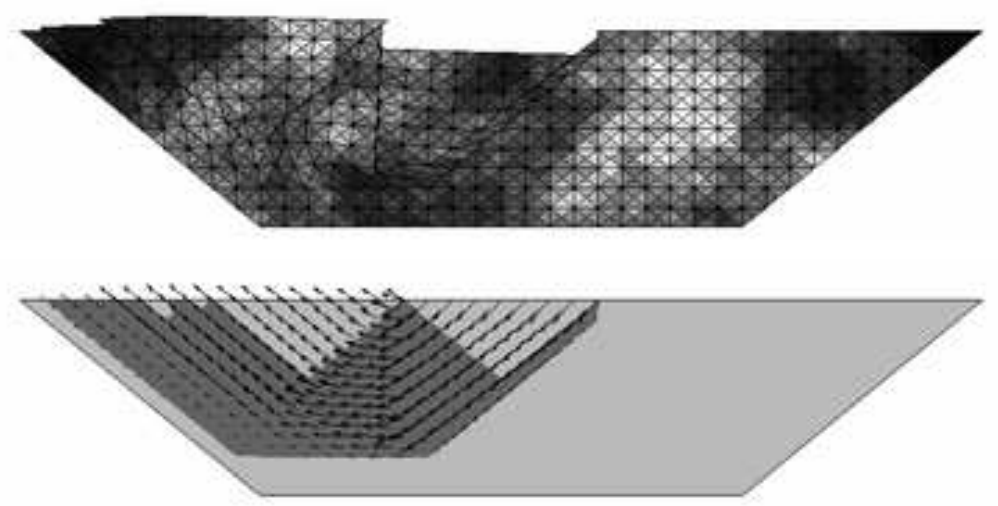

c) $\operatorname{COV}_{x_{s}=0.4,} \Theta_{\ln s_{s}-2}$

Fig. 3. Typical results of Upper Bound numerical limit analyses 


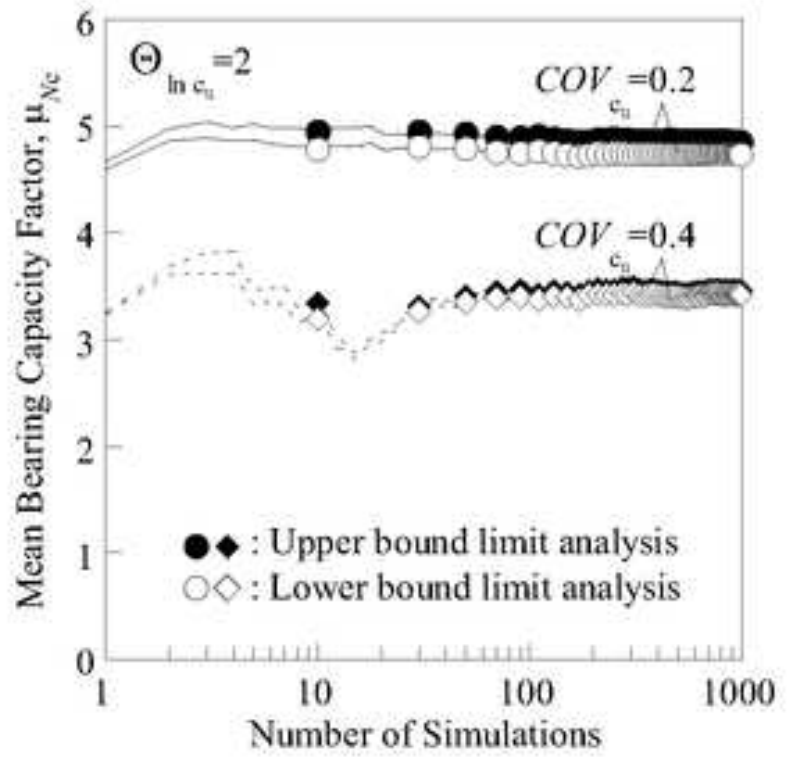

a) Mean bearing capacity factor

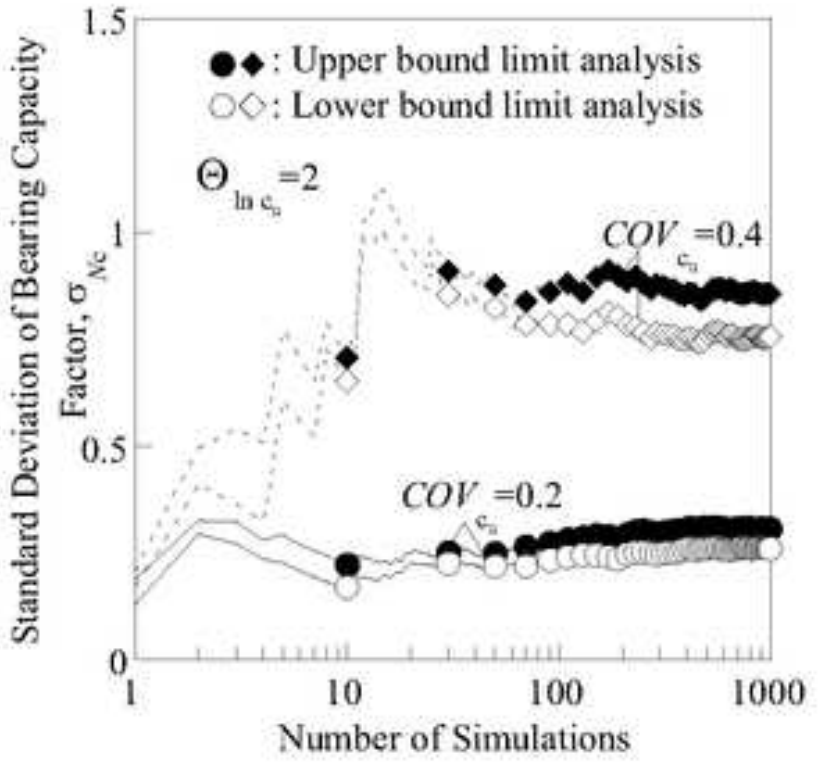

b) Standard deviation of bearing capacity factor

Fig. 4. Summary statistics of bearing capacity factor as functions of the number of Monte-Carlo simulations 


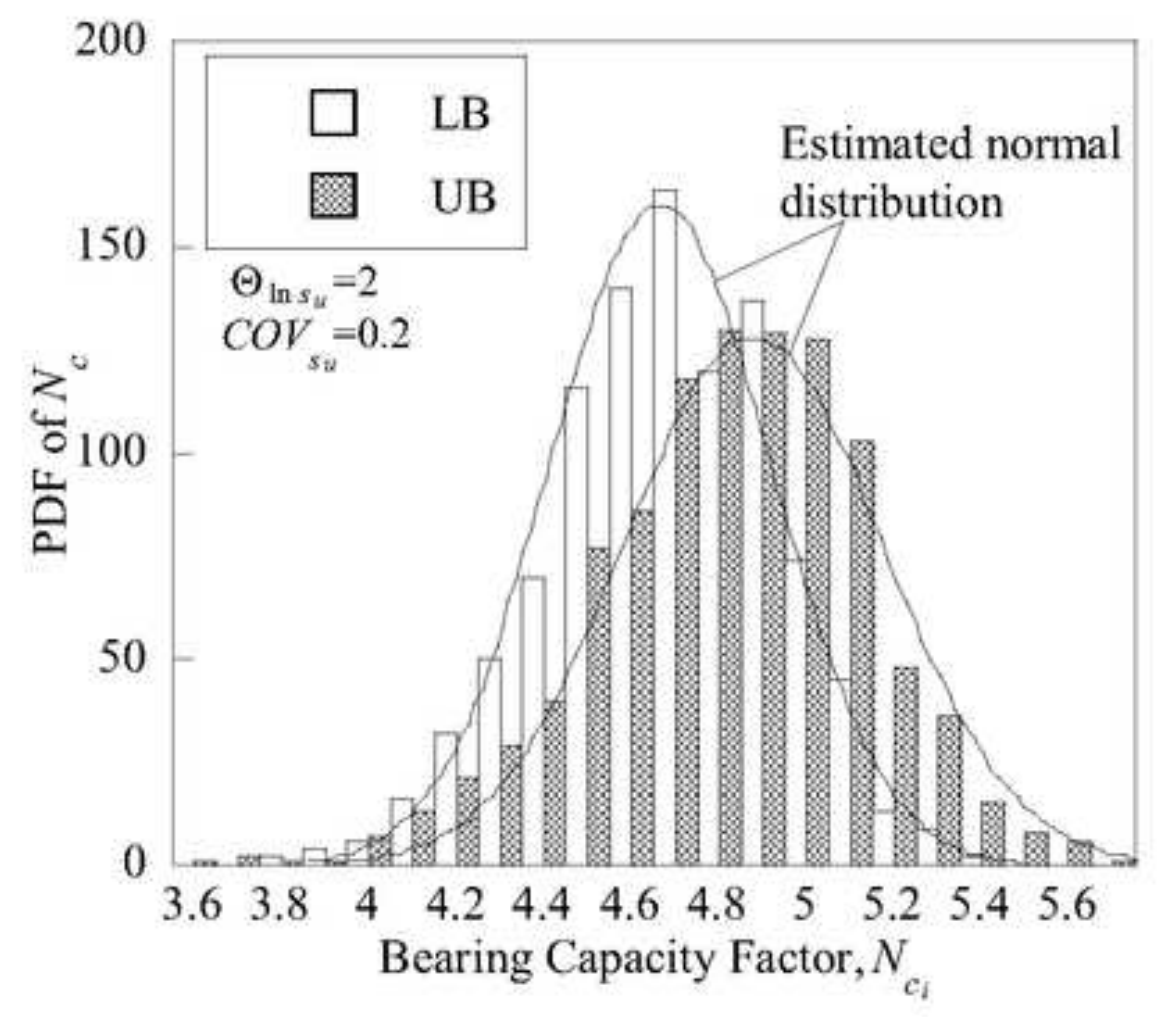

Fig. 5. Histogram and estimated normal distribution of bearing capacity factor 


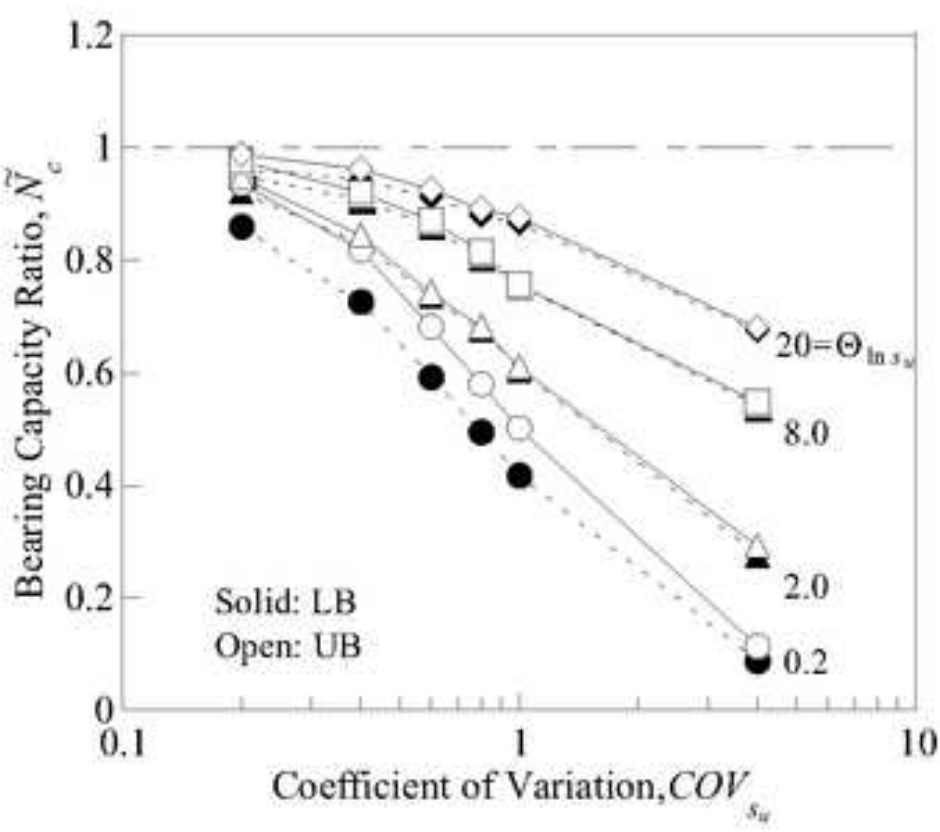

a) function of $\mathrm{COV}_{s_{\mathrm{s}}}$

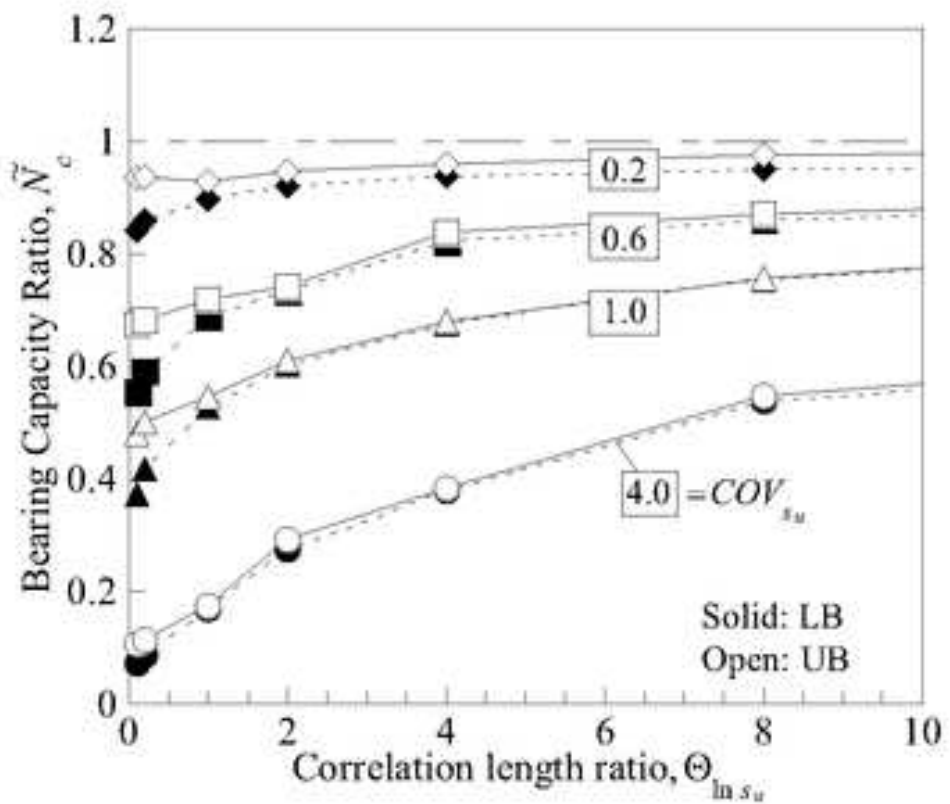

b) function of $\Theta_{\mathrm{lhs}}$

Fig. 6. Summary of mean bearing capacity ratio $\tilde{N}_{c}$ 


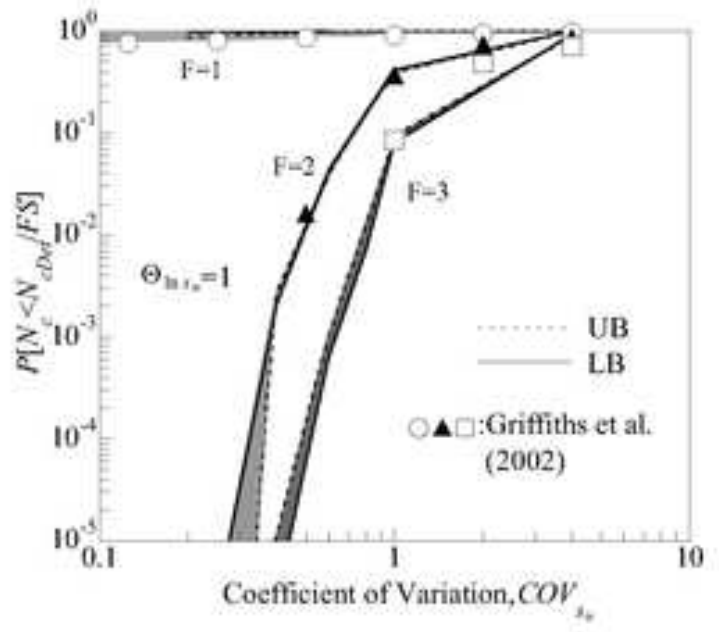

a) $\Theta_{\ln 6}=1$

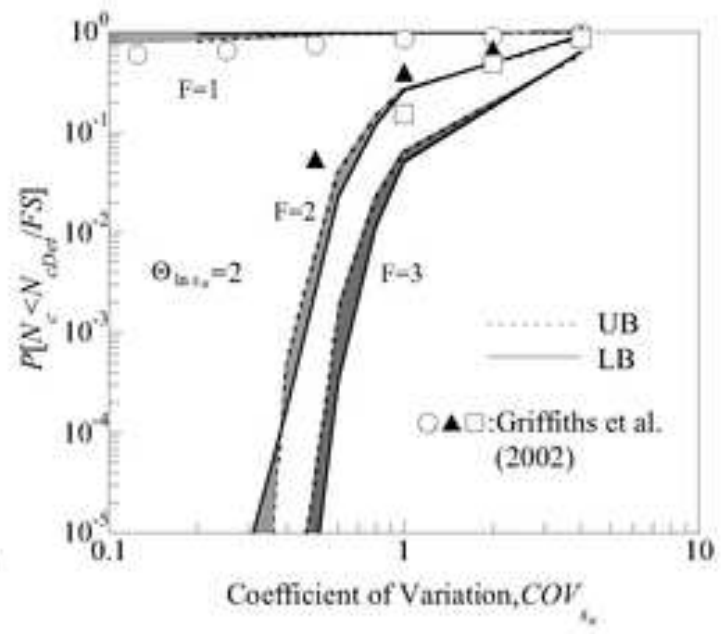

b) $\Theta_{h,}=2$

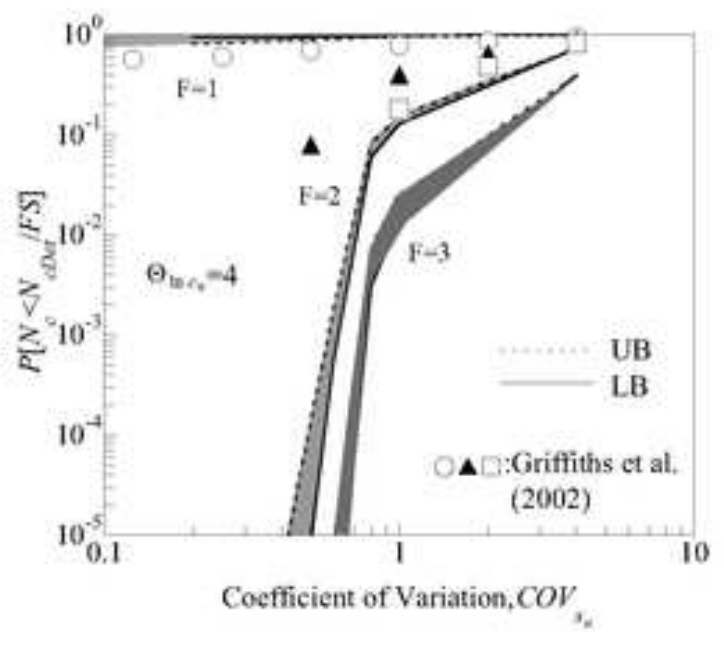

c) $\Theta_{\mathrm{hs},}=4$

Fig. 7. Probability that the bearing capacity factor is less than the nominal design load

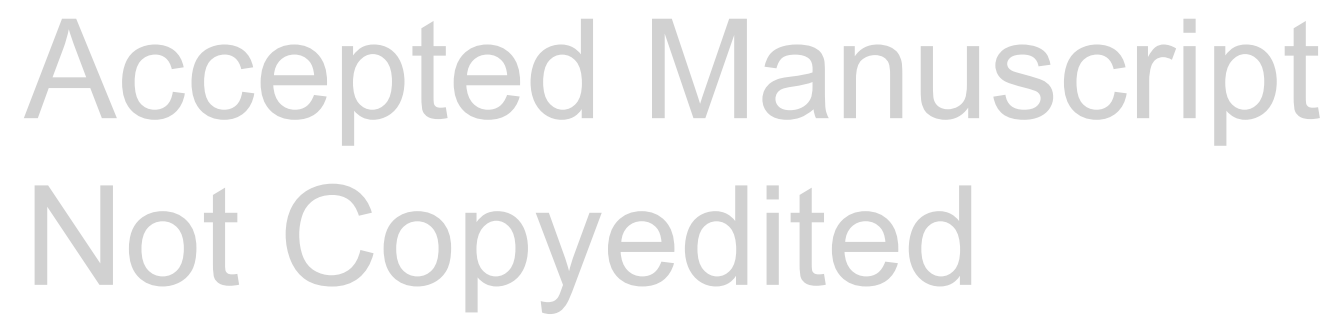




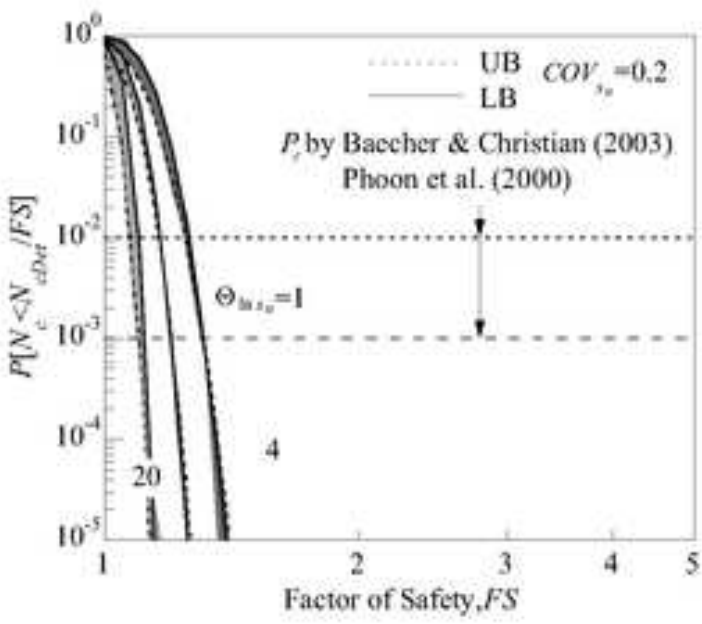

a) $\mathrm{COV}_{t_{s}}=0.2$

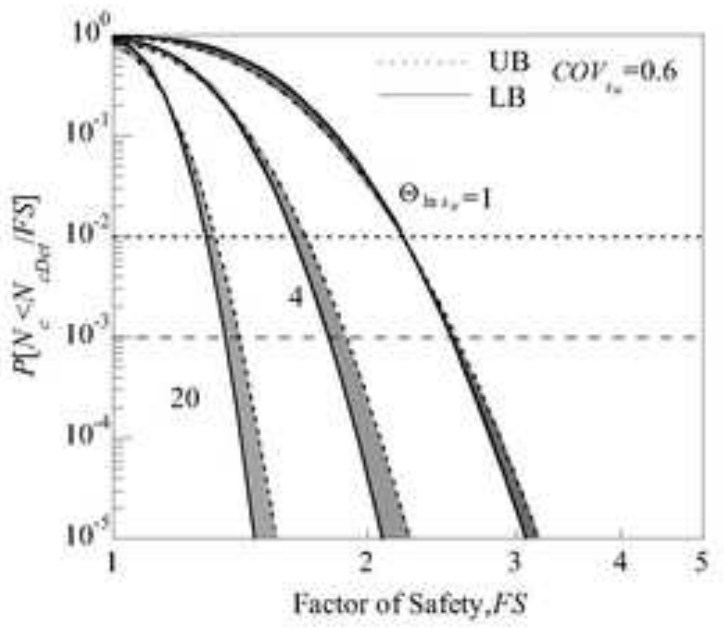

c) $\mathrm{COV}_{s_{*}}=0.6$

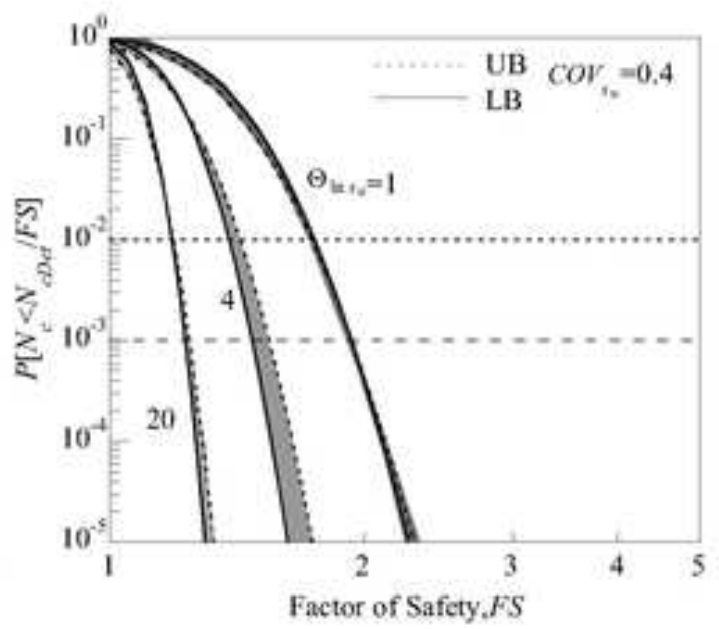

b) $\operatorname{COV}_{x_{0}}=0.4$

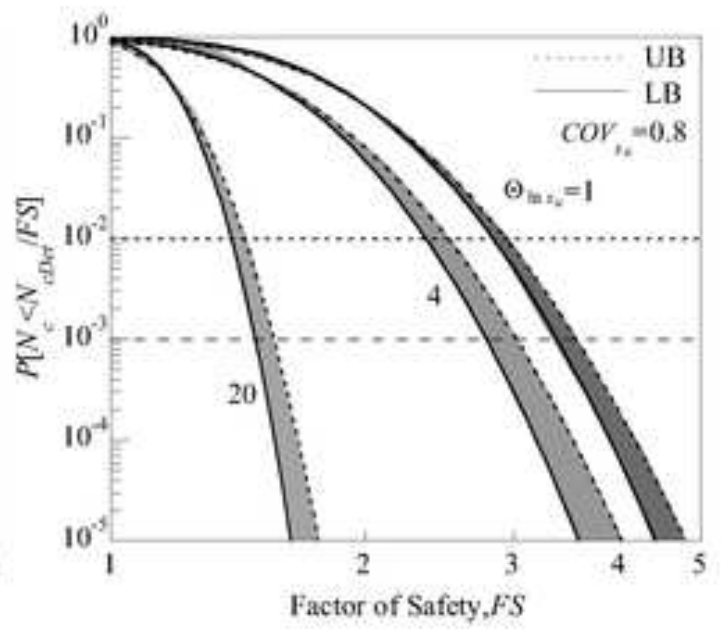

d) $\mathrm{COV}_{\mathrm{s}_{\mathrm{s}}}=0.8$

Fig. 8. Comparison of failure probability and conventional safety factor

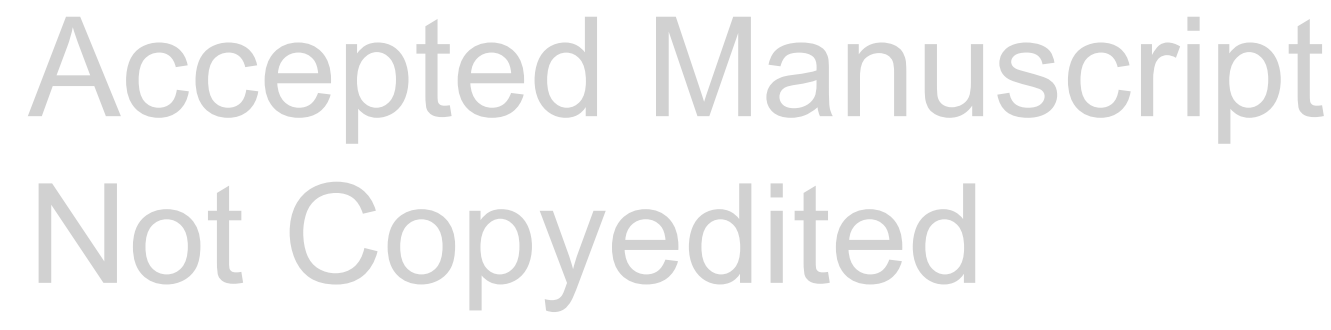

